\title{
Twin-Grating Fiber Optic Sensors Applied on Wavelength-Division Multiplexing and Its Numerical Resolution
}

José Trinidad Guillen Bonilla, Héctor Guillen Bonilla, Antonio Casillas Zamora, Gustavo Adolfo Vega Gómez, Nancy Elizabeth Franco Rodríguez, Alex Guillen Bonilla and Juan Reyes Gómez

Additional information is available at the end of the chapter

http://dx.doi.org/10.5772/intechopen.75586

\begin{abstract}
In this work, the twin-grating fiber optic sensor has been applied on wavelength-division multiplexing. A quasi-distributed sensor formed by three local twin-grating sensors, is numerically simulated. The wavelength channels were 1531.5, 1535.5, and $1539.5 \mathrm{~nm}$. The numerical simulation shows the resolution vs. signal-to-noise rate. Three local twingrating sensors have approximately the same resolution because all local sensors have the same cavity length and the wavelength channels are very close. All local sensors have two numerical resolutions because the Fourier domain phase analysis algorithm makes two evaluations of the Bragg wavelength shift. The transition between both resolutions can be calculated with the parameters: cavity length, Bragg wavelength channel, refraction index, and enveloped resolution. This transition depends on the noise system, demodulation algorithm, instrumentation, and local sensor properties. A very important point is, a theoretical analysis will permit to know the exact resolution for each local twingrating sensor.
\end{abstract}

Keywords: twin-grating fiber optic sensor, wavelength-division multiplexing, numerical resolution, quasi-distributed sensor, numerical simulation 


\section{Introduction}

Optic fiber sensors (OFSs) exhibit small dimensions; they are light weight and made of a dielectric material, vitreous silica. Some measurable parameters are temperature, strain, humidity, pressure, salinity, current, voltage, and concentration. Fiber sensors have as good resolution and accuracy as electronic and mechanical sensors. For this reason, OFSs are very active worldwide. An optic fiber sensor can be extrinsic or intrinsic. In an extrinsic sensor, the fiber acts as a means of getting the light to the sensing localization. In an intrinsic sensor, perturbations act on the fiber and the fiber in turn changes some characteristics of the light inside the fiber [1]. Both sensors types find potential industrial applications. On the other hand, a fiber sensor can also be spatially classified as a distributed sensor, a quasi-distributed sensor, or a point sensor. A distributed sensor is sensitive along its entire length. A quasi-distributed fiber optic sensor is not sensitive along its entire length, but is locally sensitized at various points. A point sensor is sensitive at a specific point along its entire length. In particular, a quasi-distributed sensor uses multiplexing techniques and their combinations. Two fundamental techniques are wavelength-division multiplexing (WDM) and frequency-division multiplexing (FDM). In Ref. [2], Grattan and Sun described the WDM technique:

- The WDM technique received little attention due to the initial high cost of components such as wavelength selective couplers and filters. However, the widespread use of Bragg grating systems has opened up a range of possibilities for the use of wavelength-division multiplexing. Figure 1a illustrates a scheme of a quasi-distributed sensor based on the Bragg gratings; its configuration is serial and each Bragg grating has its own Bragg wavelength.

The frequency-division multiplexing scheme [3] is illustrated in Figure $\mathbf{1 b}$ for a quasidistributed sensor based on the twin-grating fiber optic sensor. Each twin-grating sensor [4, 5] consists of two identical Bragg gratings and acts as a local sensor. In this configuration, there are $m$-twin-grating sensors in serial connection. Each interferometer has its own cavity length. However, all Bragg gratings have the same Bragg wavelength to eliminate wavelengthdivision multiplexing (WDM). The cross-talk noise is eliminated because all Bragg grating had low reflectivity, $r<1 \%$ [5]. In this sensor, the reflection spectrum is the superposition of all frequency components which are produced by all local interferometers. The detection method is known as direct spectrometric detection technique.

Nowadays, the quasi-distributed sensor finds potential application in civil engineering (strain and temperature measurements), industrial process (temperature, strain, level, and pressure measurements), military application (vibration detection), sport science (vibration and strain), and aircraft (strain, vibration, and pressure measurements) [6-9]. This sensor type reduces the cost by sensing point. In this work, a quasi-distributed sensor based on wavelength-division multiplexing and twin-grating sensor is discussed and simulated. The results show the numerical resolution in terms of Bragg wavelength shift. The results demonstrate that twin-grating sensors' resolution is high and the resolution depends on the cavity length. 


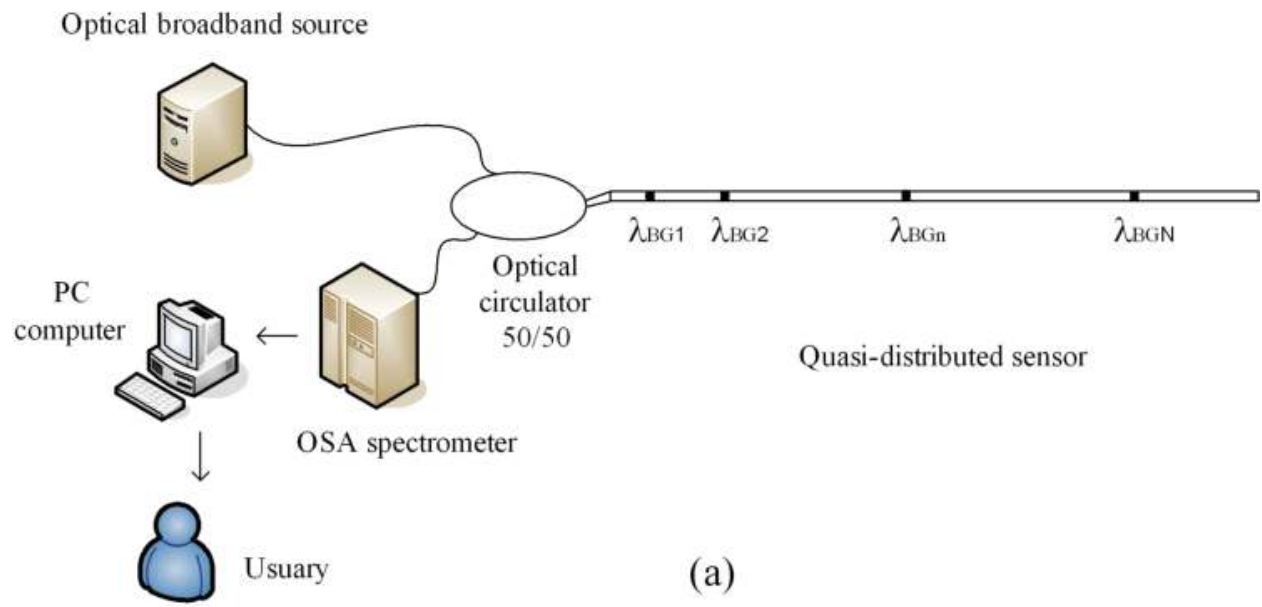

Optical broadband source

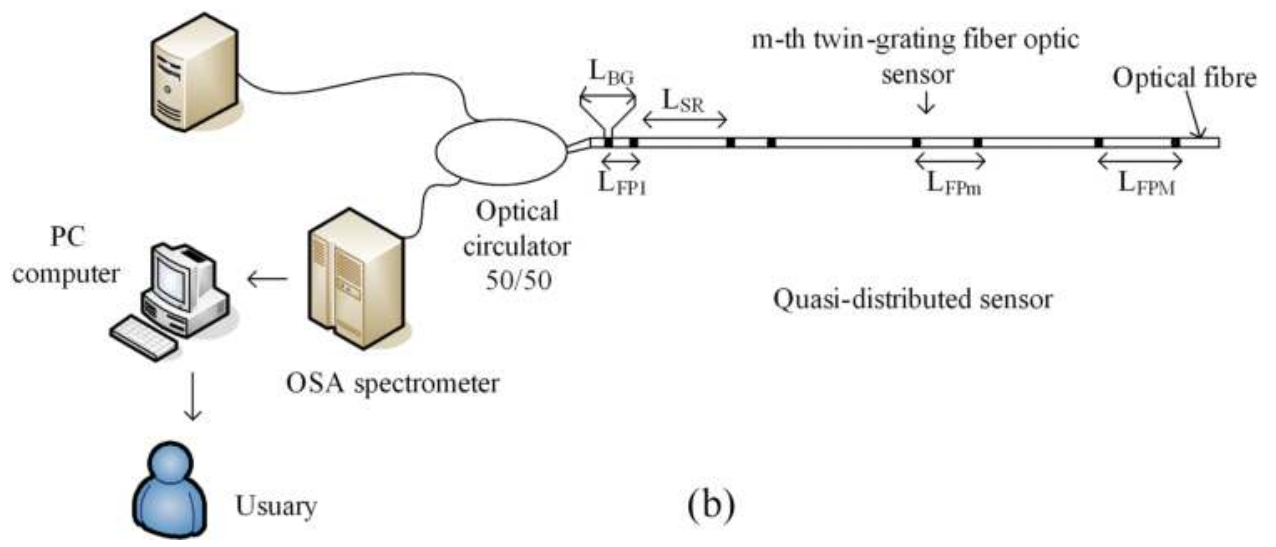

Figure 1. A scheme of a quasi-distributed fiber optic sensor: (a) Bragg gratings and (b) twin-grating interferometers [3].

\section{A quasi-distributed fiber optic sensor}

Figure 2 illustrates the optical system under study. The optic system consists of a quasidistributed fiber optic sensor which is based on wavelength-division multiplexing (WDM) and twin-grating sensors. The sensing system has five fundamental components: an optical broadband source, an optical circulator 50/50, an optical spectrometer analyzer (OSA spectrometer), a personal computer, and a quasi-distributed sensor. In particular, the quasi-distributed sensor 


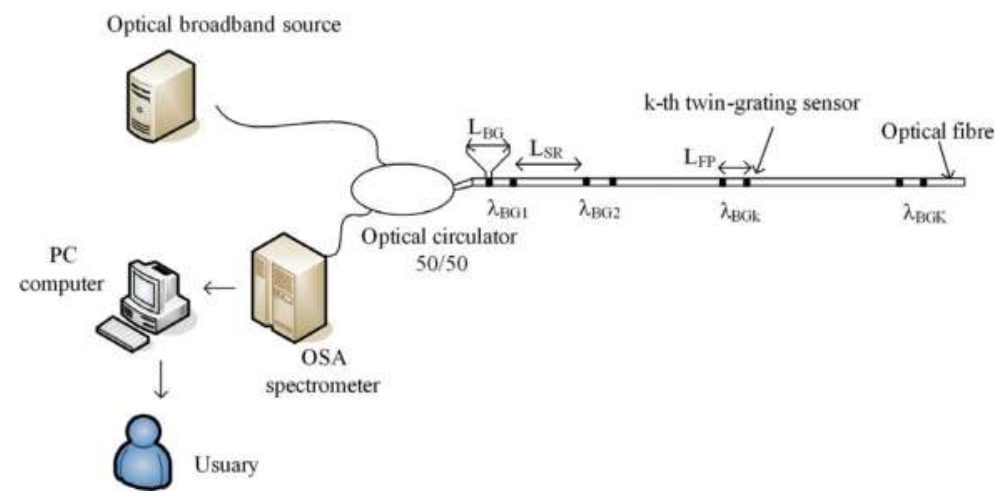

Figure 2. A quasi-distributed sensor based on the WDM technique and twin-grating sensors.

consists of a serial array of twin-grating sensors. Each twin-grating sensor acts as a low-finesse Fabry-Perot interferometer [3,10]. All interferometers have the same cavity length $L_{F P}$ but each twin-grating sensor has its own Bragg wavelength. Thus, the WDM technique is generated and the FDM technique is eliminated. All Bragg gratings have the same length $L_{B G}$ and a typical reflectivity of $1 \%$. The low reflectivity eliminates cross-talk noise.

\subsection{Optical signal}

In the sensing system presented in Figure 2, the signal from each local sensor is returned by reflection from each twin-grating sensor, where each twin-grating interferometer has its own wavelength. The signal returned to the detector is monitored with the OSA spectrometer; the intensity at each wavelength corresponds to the measurement each local sensor. When the quasi-distributed sensor does not have external perturbations and interference patterns have small variation, the optical signal will be

$$
R_{T}(\lambda)=\sum_{k=1}^{K} 2 a_{k}\left[\left(\frac{\pi n_{1} L_{B G}}{\lambda_{B G k}}\right)^{2} \operatorname{sinc}^{2}\left(\frac{2 n_{1} L_{B G}\left(\lambda-\lambda_{B G k}\right)}{\lambda_{B G k}^{2}}\right)\right]\left[1+\cos \left(\frac{4 \pi n L_{F P}\left(\lambda-\lambda_{B G k}\right)}{\lambda_{B G k}^{2}}\right)\right]
$$

The signal parameters are: $R_{T}(\lambda)$ is a set of interference patterns, $\lambda$ is the wavelength, $\lambda_{B G k}$ is the $k$ th Bragg wavelength, $a_{k}$ are amplitude factors, $n_{1}$ is the amplitude of the effective refractive index modulation of the gratings, $L_{B G}$ is the length of gratings, $n$ is the effective index of the core, $L_{F P}$ is the cavity length, and $k$ is the number of twin-grating sensors. The optical signal has the next characteristics: the enveloped function is a sinc one and its width $\Delta_{B G k}$ is defined as the spectral distance between its +1 and -1 zeros,

$$
\Delta_{B G 1} \neq \Delta_{B G 2} \neq \Delta_{B G 3} \neq \ldots \neq \Delta_{B G k}=\frac{\lambda_{B G k}^{2}}{n_{1} L_{B G}} \neq \ldots \neq \Delta_{B G K}
$$

Each interference pattern has its own central Bragg wavelength and the next condition is true 


$$
\lambda_{B G 1} \neq \lambda_{B G 2} \neq \ldots \neq \lambda_{B G k} \neq \ldots \neq \lambda_{B G K}
$$

Interference patterns have approximately the same frequency,

$$
v_{F P 1} \approx v_{F P 2} \approx \ldots \approx v_{F P k}=\frac{2 n L_{F P}}{\lambda_{B G k}^{2}} \approx \ldots \approx v_{F P K}
$$

From Eqs. (1) and (4), the cavity length defines the frequency of all interference patterns. Its size can be found in the interval of [3]

$$
L_{F P \min } \leq L_{F P} \leq L_{F P \max } \rightarrow 2 L_{B G} \leq L_{F P} \leq \frac{\lambda_{B G 1}^{2}}{4 n \Delta \lambda}
$$

where $\Delta \lambda$ is the spectrometer resolution, $L_{F P \min }=2 L_{B G}$ is the minimum cavity length, and $L_{F P \max }=\frac{\lambda_{B G 1}^{2}}{4 n \Delta \lambda}$ is the maximum cavity length. The minimum cavity length is delimited because the Fourier domain phase analysis (FDPA) algorithm does not accept additional information or loss of information. The maximum cavity length is delimited because the OSA spectrometer has a limit of full-width half-maximum (FWHM). Figure 3 illustrates the optical spectrum.

To know the frequency spectrum $R(v)$, the Fourier transform is applied to Eq. (1),

$$
R_{T}(v)=\int_{-\infty}^{\infty} R_{T}(\lambda) e^{-i 2 \pi \lambda v} d \lambda
$$

Substituting Eq. (1) into Eq. (6), the spectra $R_{T}(v)$ is

$$
R(v)=\int_{-\infty}^{\infty} \sum_{k=1}^{K} 2 a_{k}\left[\left(\frac{\pi n_{1} L_{B G}}{\lambda_{B G k}}\right)^{2} \operatorname{sinc}^{2}\left(\frac{2 n_{1} L_{B G}\left(\lambda-\lambda_{B G k}\right)}{\lambda_{B G k}^{2}}\right)\right]\left[1+\cos \left(\frac{4 \pi n L_{F P}\left(\lambda-\lambda_{B G k}\right)}{\lambda_{B G k}^{2}}\right)\right] e^{-i \omega t} d t
$$

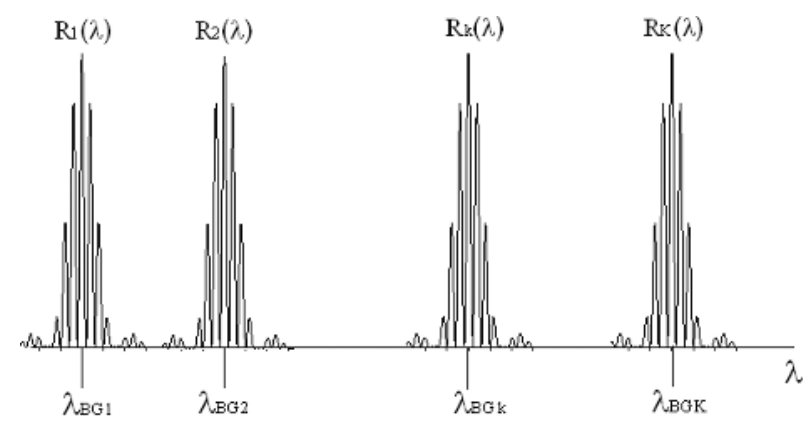

Figure 3. Optical signal detected by the optical analyzer spectrometer. 
Solving the transformation, the frequency spectrum is defined by

$$
R_{T}(v)=\sum_{k=-K}^{K} R_{k}(v)=\sum_{k=-K}^{K} c_{k} t r i\left(\frac{v-v_{F P k}}{v_{B G k}}\right)
$$

This frequency spectrum is the superposition of a set of triangle functions, where a triangle function is defined as $\operatorname{tri}(x)=\left\{\begin{array}{cc}1-|x| & |x| \leq 1 \\ 0 & \text { otherwise }\end{array}, R_{k}(v)\right.$ is the Fourier transform of the $k$-th interference pattern, $c_{k}$ are amplitude factors, $v_{F P k}$ is the center position of each peak and $v_{B G k}$ is the bandwidth of each peak.

$$
v_{B G k}=\frac{4 n_{1} L_{B G}}{\lambda_{B G k}^{2}}
$$

In the frequency spectrum, the component $v_{F P 0}$ contains information from all twin-grating sensors, the positive components $v_{F P 1}, \ldots, v_{F P K}$; the negative components $-v_{F P 1}, \ldots,-v_{F P K}$ contain the same information. The minimum bandwidth $v_{B G \min }$ is

$$
v_{B G \min }=\frac{4 n_{1} L_{B G}}{\lambda_{B G K}^{2}}
$$

and the maximum bandwidth $v_{B G \max }$ is

$$
v_{B G \max }=\frac{4 n_{1} L_{B G}}{\lambda_{B G 1}^{2}}
$$

Figure 4 shows the frequency spectrum $R(v)$. Based on Figure 4, all twin-grating interferometers produce approximately the same frequency components. This is possible because all interferometers have the same cavity length and all enveloped functions are approximately similar.

\subsection{Optical signal produced by external perturbation}

When the quasi-distributed sensor has external perturbations due to the temperature or strain, Bragg gratings and cavity length have an elongation. In turn, interference patterns have a small shift in response to a measured variation. The optical signal detected by the OSA spectrometer is

$$
\begin{aligned}
R_{T}(\lambda, \delta \lambda)= & \sum_{k=1}^{K} 2 a_{k}\left[\left(\frac{\pi n_{1} L_{B G}}{\lambda_{B G k}}\right)^{2} \operatorname{sinc}^{2}\left(\frac{2 n_{1} L_{B G}\left(\lambda-\lambda_{B G k}-\delta \lambda_{k}\right)}{\lambda_{B G k}^{2}}\right)\right] \\
& {\left[1+\cos \left(\frac{4 \pi n L_{F P}\left(\lambda-\lambda_{B G k}-\delta \lambda_{k}\right)}{\lambda_{B G k}^{2}}\right)\right] }
\end{aligned}
$$

It can also be expressed as 


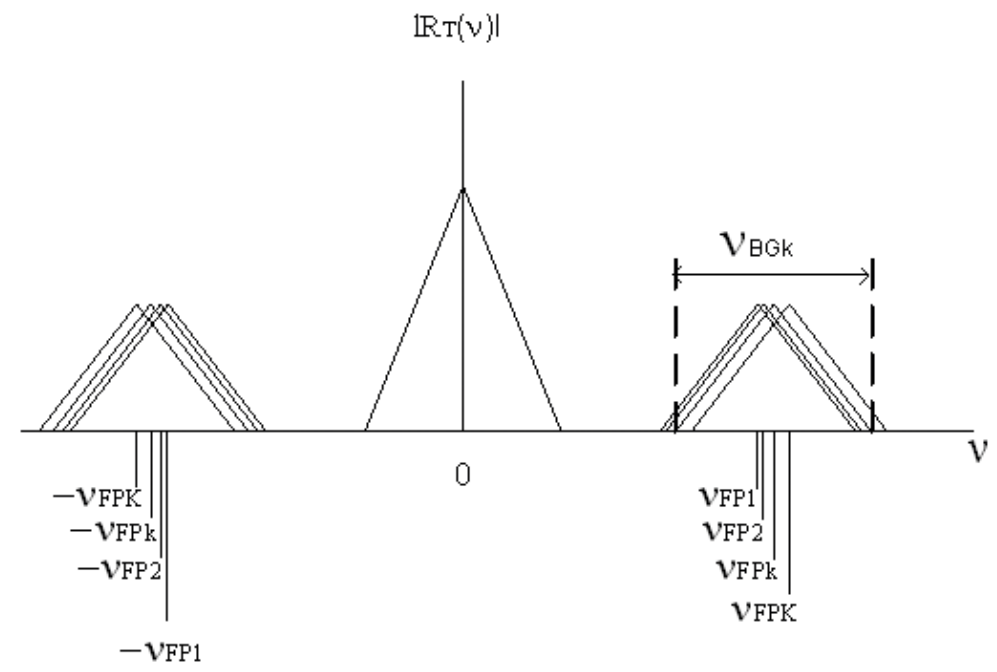

Figure 4. Frequency spectrum determined from the optical signal.

$$
R_{T}(\lambda, \delta \lambda)=\sum_{k=1}^{K} R_{k}\left(\lambda-\delta \lambda_{k}\right)=R_{1}\left(\lambda-\delta \lambda_{1}\right)+\ldots+R_{k}\left(\lambda-\delta \lambda_{k}\right)+\ldots+R_{K}\left(\lambda-\delta \lambda_{K}\right)
$$

where $R_{T}(\lambda, \delta \lambda)$ is the optical signal due to external perturbations and $\delta \lambda_{k}$ is the Bragg wavelength shift due to measured change [11, 12]. Its frequency spectrum $R_{T}(v, \delta \lambda)$ is

$$
R_{T}(v, \delta \lambda)=\int_{-\infty}^{\infty} R_{T}(\lambda, \delta \lambda) e^{-i 2 \pi \lambda v} d \lambda
$$

Substituting Eq. (13) into Eq. (14), the spectra is now

$$
R_{T}(v, \delta \lambda)=\int_{-\infty}^{\infty} \sum_{k=-K}^{K} R_{k}\left(\lambda-\delta \lambda_{k}\right) e^{-i 2 \pi \lambda v} d \lambda
$$

Using the Fourier transform properties and solving, the frequency spectra $R_{T}(v, \delta \lambda)$ takes the form

$$
R_{T}(v, \delta \lambda)=\sum_{k=-K}^{K} e^{-i 2 \pi v \delta \lambda_{k}} R_{k}(v)
$$

$R_{T}(v, \delta \lambda)$ is the multiplication between $R(v)$ and a set of phases. Each phase contains information of each twin-grating sensor and then the FDPA algorithm can be applied in the demodulation signal. 


\section{Number of samples}

In Ref. [11] the twin-grating sensor was applied for the temperature measurement. The wavelength shift sensitivity to a temperature change was estimated to be $0.00985 \mathrm{~nm} /{ }^{\circ} \mathrm{C}$. The demodulation signal was done using the Fourier domain phase analysis algorithm. The optical signal was acquired applying direct spectrometric detection. This detection technique uses an optical spectrometer analyzer; then, the acquired optical signal becomes discrete. The signal samples $R_{T}\left(\lambda_{j}\right)$ are taken as wavelengths $\lambda_{j}=\lambda_{\min }+j \delta \lambda_{s}$, where $j=0,1, \ldots, N-1, N$ is the number of samples. The interval working is $\lambda_{w}=\lambda_{\max }-\lambda_{\min }: \lambda_{\max }$ is the maximum wavelength, $\lambda_{\min }$ is the minimum wavelength and $\delta \lambda_{s}$ is the wavelength step.

From Figure 4, the maximum frequency $v_{\max }$ is

$$
v_{\max }=v_{F P K}+\frac{1}{2} v_{B G \max }
$$

Substituting Eqs. (4)-(11) into Eq. (17), the maximum frequency is

$$
v_{\max }=\frac{2 n L_{F P \max }}{\lambda_{B G 1}^{2}}+\frac{2 n_{1} L_{B G}}{\lambda_{B G 1}^{2}}
$$

When we substitute the maximum cavity length (Eq. (5)) into Eq. (18), the parameter $v_{\max }$ takes the form

$$
v_{\max }=\frac{1}{2 \Delta \lambda}+\frac{2 n_{1} L_{B G}}{\lambda_{B G 1}^{2}}
$$

Applying the sampling theorem, the sampling frequency $v_{s}$ is

$$
v_{s} \geq 2 v_{\max }=\frac{1}{\Delta \lambda}+\frac{4 n_{1} L_{B G}}{\lambda_{B G 1}^{2}}
$$

Since $v_{s}=\frac{1}{\delta \lambda_{s}}$, we have

$$
\delta \lambda_{s} \leq \frac{\Delta \lambda \lambda_{B G 1}^{2}}{\lambda_{B G 1}^{2}+4 n_{1} L_{B G} \Delta \lambda}
$$

Finally, the number of samples $N$ is given by

$$
N=\frac{\lambda_{w}}{\delta \lambda_{s}}=\frac{\lambda_{w}\left(\lambda_{B G 1}^{2}+4 n_{1} L_{B G} \Delta \lambda\right)}{\Delta \lambda \lambda_{B G 1}^{2}}
$$

Samples $N$ depend on twin-grating sensor properties, the spectrometer resolution, and the interval working. The number of samples is a very important parameter for the twin-grating sensor demodulation because it affects the sensor's resolution. 


\section{Capacity of wavelength-division multiplexing}

In Refs. [4, 5] two experimental sensing systems where twin-grating fiber optic sensors were applied on wavelength-division multiplexing were reported. The first optical system consisted of two wavelength channels. Both channels were centered around 815 and $839 \mathrm{~nm}$. The second optical system consisted of three wavelength channels. The channels were around 1542, 1548, and $1554 \mathrm{~nm}$. Therefore, based on the Bragg grating characteristics, the twin-grating interferometer can be applied in wavelength-division multiplexing if and only if each interferometer sensor has its own Bragg wavelength: $\lambda_{B G k}=2 n_{1} \Lambda_{k}$, where $\Lambda_{k}$ is the period [1]. In this case, each interference pattern has its own bandwidth in the wavelength domain. The patterns are in the interval of $\lambda_{\min }$ until $\lambda_{\max }$ (interval working $\lambda_{w}$ ); it is not possible in other positions, see Figure 5.

Let us introduce the operation range $\Delta \lambda_{\mathrm{op}}$; the operation range defines the interval in which an interference pattern can move into the wavelength domain. Each interferometer sensor has its own operation range and overlapping is not acceptable. To calculate the capacity of wavelengthdivision multiplexing $K$, we use the interval working $\lambda_{w}$ and the operation range $\Delta \lambda_{\mathrm{op}}$

$$
K=\frac{\lambda_{w}}{\Delta \lambda_{\mathrm{op}}}=\frac{\lambda_{\max }-\lambda_{\min }}{\Delta \lambda_{\mathrm{op}}}
$$

$K$ is the number of local sensors and wavelength channels. $\lambda_{w}, \lambda_{\min }, \lambda_{\max }$, and $\Delta \lambda_{\mathrm{op}}$ parameters can be observed in Figure 3.

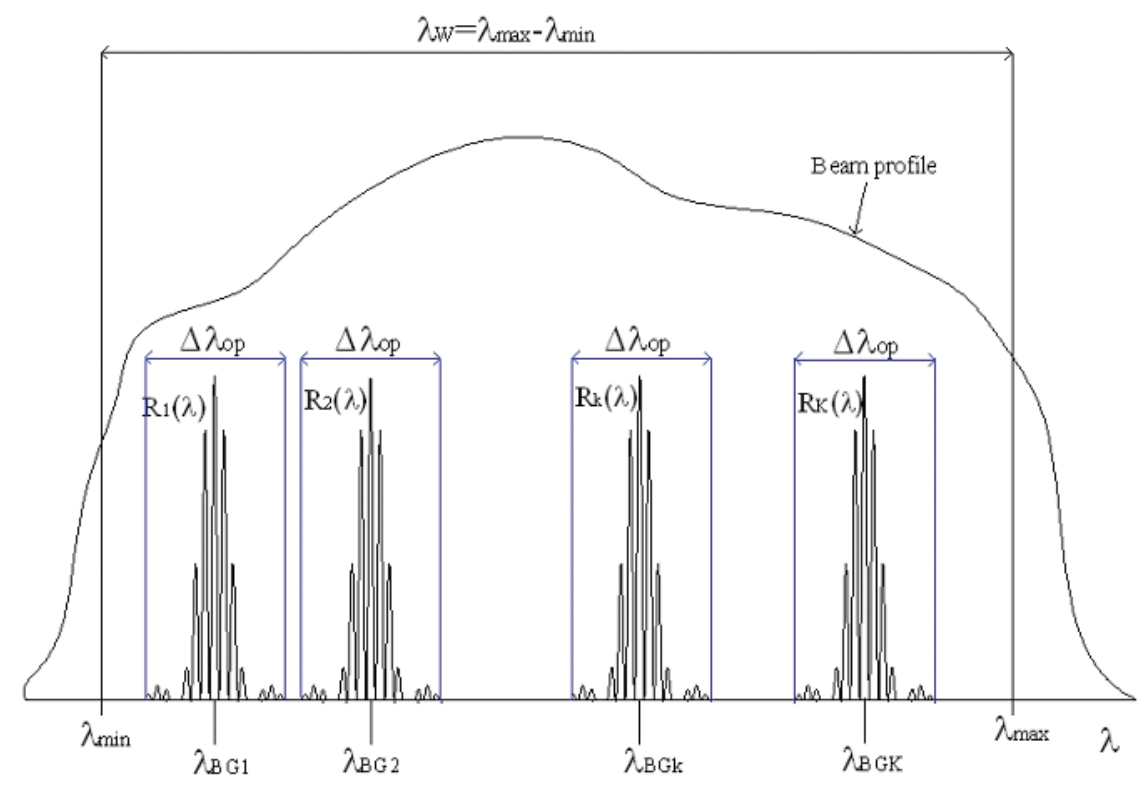

Figure 5. $\lambda_{w}, \lambda_{\min }, \lambda_{\max }$, and $\Delta \lambda_{o p}$ representation. 
To illustrate, the next numerical example is presented.

Example 1: A broadband light source has the interval from $\lambda_{\min }=1470 \mathrm{~nm}$ to $\lambda_{\max }=1620 \mathrm{~nm}$. If the operation range is selected to $\Delta \lambda_{\mathrm{op}}=6 \mathrm{~nm}$, the number of local sensors is $K=\frac{1620-1470}{6}$ $=25$. The quasi-distributed sensor would have 25 twin-grating sensors and the signal will have 25 wavelength channels. Here, two important points can be mentioned: 1) The parameter $\Delta \lambda_{\text {op }}$ permits the selection of the number of local sensors; 2 ) the cost per sensing point can be reduced combining the wavelength-and-frequency division multiplexing.

\section{Demodulation signal}

In this section, we present the demodulation signal for the quasi-distributed sensor based on wavelength-division multiplexing. The signal processing combines the Fourier domain phase analysis (FDPA) algorithm, a bank of $K$ filters and a band-pass filter. The FDPA algorithm was described and also applied in Refs. [3, 11]. The bank of $K$ filters can be defined as

$$
\mathrm{F}(\lambda)=\operatorname{rect}\left(\frac{\lambda}{\Delta \lambda_{\mathrm{op}}}\right) \otimes \sum_{k=1}^{K} \delta\left(\lambda-\lambda_{B G k}\right)
$$

where the symbol $\otimes$ indicates the convolution operation, the rect function (Eq. (24)) is defined as

$$
\operatorname{rect}(\lambda)=\left\{\begin{array}{cc}
1 & |\lambda|<\frac{\Delta \lambda_{\mathrm{op}}}{2} \\
0 & |\lambda|>\frac{\Delta \lambda_{\mathrm{op}}}{2}
\end{array}\right.
$$

and $\delta$ is the Dirac delta. Invoking the Dirac delta properties, the bank of $K$ filters is

$$
\mathrm{F}(\lambda)=\sum_{k=1}^{K} \operatorname{rect}\left(\frac{\lambda-\lambda_{B G k}}{\Delta \lambda_{\mathrm{op}}}\right)
$$

The signal $F(\lambda)$ is a series of rect functions in the wavelength domain; its bandwidth is the operation range and the central positions are the Bragg wavelengths. On the other hand, $R_{k}(v)$ is the Fourier transform for the $k$ th interference pattern. The spectrum $R_{k}(v)$ consists of three triangle functions. The component $v_{F P 0}$ contains information from all twin-grating sensors and this signal cannot be used in the demodulation signal. The $v_{F P k}$ and $-v_{F P k}$ components contain the same information and any component can be used in the demodulation signal. Then, the band-pass filter can be defined as

$$
\mathrm{F}(v)=\operatorname{rect}\left(\frac{v-v_{F P k}}{v_{B G k}}\right)
$$

where the rect function (Eq. (27)) has the next definition 


$$
\operatorname{rect}(v)= \begin{cases}1 & |v|<\frac{v_{B G k}}{2} \\ 0 & |v|>\frac{v_{B G k}}{2}\end{cases}
$$

The filter $\mathrm{F}(v)$ is a rect function in the frequency domain: its bandwidth is $v_{B G k}$ and its central position is $v_{F P k}$.

Basically, the digital demodulation consists of two stages: calibration and measurement. The calibration stage is developed once; five steps are necessary and the references are generated. The calibration considers the signal acquisition $R_{T}(\lambda)$, filtering signal $R_{k}(\lambda)$, separation of frequency components $R_{k}(v)$, filtering $R_{m}(\lambda)$, and its complex conjugate $R_{m}^{*}(\lambda)$, where the symbol * indicates the complex conjugate. The measurement stage is developed for each measurement and eight steps are necessary. The measurement stage considers the signal acquisition $R_{T}(\lambda, \delta \lambda)$, filtering $R_{k}(\lambda, \delta \lambda)$, separation of frequency components $R_{T}(v, \delta \lambda)$, filtering $R_{m}(v, \delta \lambda)$, comparison between spectrums $R_{m}^{*}(\lambda)$ and $R_{m}(v, \delta \lambda), 2 \pi P$ ambiguity elimination; Bragg wavelength shift evaluation and an adaptive filter is applied. The adaptive filter is a set of coefficients as was described in Ref. [13]. The complete procedure is shown at Figure 6.

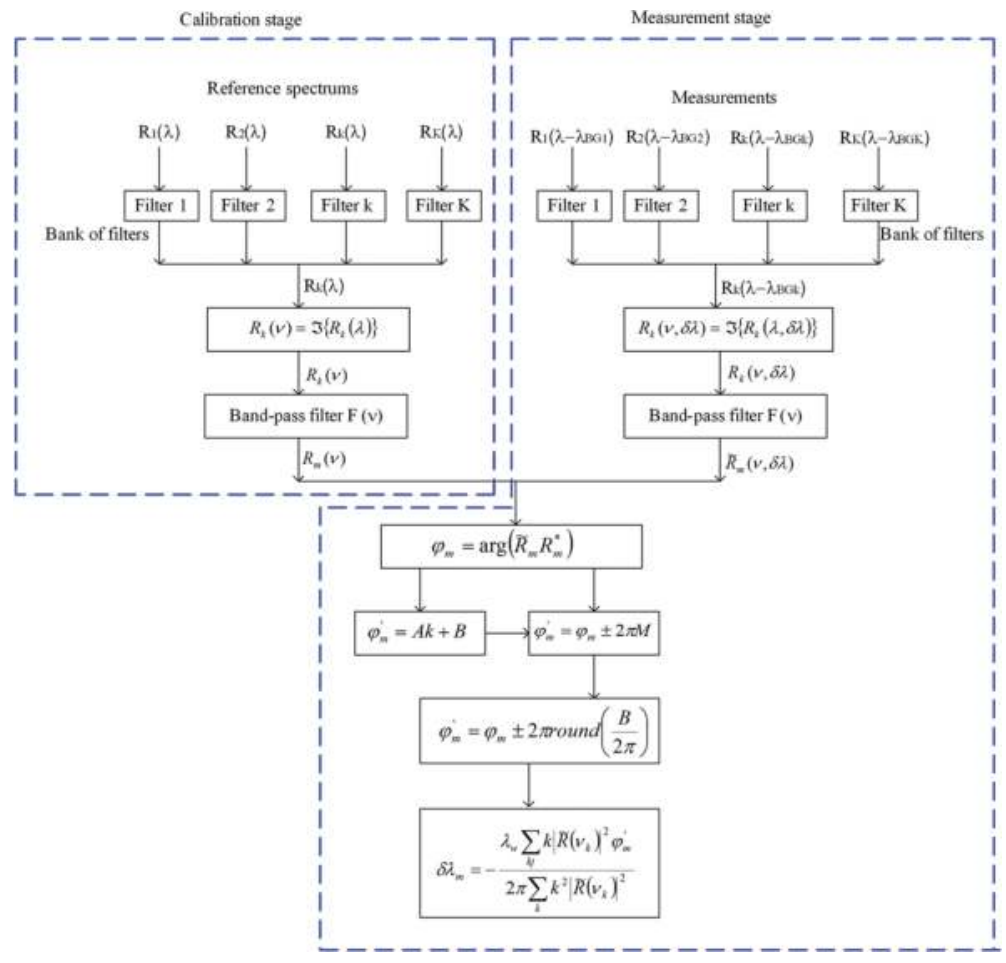

Figure 6. Digital demodulation represented schematically: $R_{m}^{*}=R_{m}^{*}(\lambda), \quad \tilde{R}_{m}=\tilde{R}_{m}(\nu, \delta \lambda)$ and the symbol * indicates the complex conjugate. 


\section{Numerical simulation and discussion}

\subsection{Parameters and results}

In Ref. [3], the twin-grating fiber optic sensor was applied on frequency-division multiplexing. The numerical results confirmed that the twin-grating sensor has a high resolution and the resolution is a function of cavity length. In the numerical simulation, the number of samples was $N=1024$, the noise was in the interval $\sqrt{S N R}=10^{0}$ to $\sqrt{S N R}=10^{4}$. The quasi-distributed sensor parameters and optical signal parameters can be observed in Table 1.

Being aware of that, our goal is to apply the twin-grating interferometer to wavelengthdivision multiplexing, a quasi-distributed fiber optic sensor is numerically simulated as was done in Ref. [3]. The quasi-distributed sensor consists of three twin-grating sensors. The physical parameters are shown in Table 2. In our numerical simulation, we use some parameters from Ref. [3]: $L_{\mathrm{FP} 1}=L_{\mathrm{FP} 2}=L_{\mathrm{FP} 3}=L_{\mathrm{FP} 4}=4(\mathrm{~mm}), L_{\mathrm{BG}}=0.5(\mathrm{~mm}), n=1.46, N=1024$; the Bragg gratings have rectangular profile; the noise has Gaussian distribution and its value is in the interval $\sqrt{S N R}=10^{0}$ to $\sqrt{S N R}=10^{4}$. The same values are possible because our goal is to prove the wavelength-division multiplexing. The measurements are in the intervals $S 1 \rightarrow-0.3$ to $0.3 \mathrm{~nm}, S 2 \rightarrow-0.5$ to $0.5 \mathrm{~nm}$ and $S 3 \rightarrow-1$ to $1 \mathrm{~nm}$. The signal parameters are shown in Table 2 while Figure 5 shows our numerical results: Demodulation errors vs. $S N R^{1 / 2}$. A Laptop Toshiba 45C was used with a $512 \mathrm{Mb}$ RAM and a velocity of $1.7 \mathrm{GHz}$.

Analyzing Tables 1 and 2, the sensing system presented in Figure 3 is based on wavelengthdivision multiplexing where their wavelength-channels are 1531.5, 1535.5, and $1539.5 \mathrm{~nm}$. The frequency-division multiplexing was eliminated since the frequency components are $v_{F P 1}=$ $4.979($ cycles $/ \mathrm{nm}), v_{F P 2}=4.953($ cycles $/ \mathrm{nm})$, and $v_{F P 3}=4.928($ cycles $/ \mathrm{nm})$. All interference patterns have approximately the same bandwidth: $\Delta \lambda_{B G 1}=3.213(\mathrm{~nm}), \Delta \lambda_{B G 2}=3.229(\mathrm{~nm})$, and

\begin{tabular}{lll}
\hline Sensor number & Sensor parameters & Signal values \\
\hline Twin-grating sensor 1 (S1) & $L_{\mathrm{FP} 1}=4(\mathrm{~mm})$ & $\Delta_{\lambda B G}=3.22(\mathrm{~nm})$ \\
& $L_{\mathrm{BG}}=0.5(\mathrm{~mm})$ & $v_{F P 1}=4.95(\mathrm{ciclos} / \mathrm{nm})$ \\
& $n=1.46$ & $v_{B G}=1.23(\mathrm{ciclos} / \mathrm{nm})$ \\
& $\lambda_{B G}=1532.5(\mathrm{~nm})$ & \\
Twin-grating sensor 2 (S2) & $L_{\mathrm{FP} 2}=8(\mathrm{~mm})$ & $\Delta_{\lambda B G}=3.22(\mathrm{~nm})$ \\
& $L_{\mathrm{BG}}=0.5(\mathrm{~mm})$ & $v_{F P 2}=9.91(\mathrm{ciclos} / \mathrm{nm})$ \\
& $n=1.46$ & $v_{B G}=1.23(\mathrm{ciclos} / \mathrm{nm})$ \\
& $\lambda_{B G}=1532.5(\mathrm{~nm})$ & \\
Twin-grating sensor 3 (S3) & $L_{\mathrm{FP} 3}=16(\mathrm{~mm})$ & $\Delta_{\lambda B G}=3.22(\mathrm{~nm})$ \\
& $L_{\mathrm{BG}}=0.5(\mathrm{~mm})$ & $v_{F P 3}=19.82(\mathrm{ciclos} / \mathrm{nm})$ \\
& $n=1.46$ & $v_{B G}=1.23(\mathrm{ciclos} / \mathrm{nm})$ \\
& $\lambda_{B G}=1532.5(\mathrm{~nm})$ & \\
\hline
\end{tabular}

Table 1. Sensor parameters and signal parameters used in the frequency-division multiplexing [3]. 


\begin{tabular}{|c|c|c|}
\hline Sensor number & Sensor parameters & Signal values \\
\hline Fabry-Perot sensor 1 (S1) & $\begin{array}{l}L_{\mathrm{FP} 1}=4(\mathrm{~mm}) \\
L_{\mathrm{BG}}=0.5(\mathrm{~mm}) \\
n=1.46 \\
\lambda_{B G 1}=1531.5(\mathrm{~nm})\end{array}$ & $\begin{array}{l}v_{F P 1}=4.979 \text { (cycles/nm) (Eq. (2)) } \\
\Delta_{\lambda B G 1}=3.213 \text { (nm) (Eq. (3)) } \\
v_{B G 1}=1.244 \text { (cycles/nm) (Eq. (9)) }\end{array}$ \\
\hline Fabry-Perot sensor 2 (S2) & $\begin{array}{l}L_{\mathrm{FP} 2}=4(\mathrm{~mm}) \\
L_{\mathrm{BG}}=0.5(\mathrm{~mm}) \\
n=1.46 \\
\lambda_{B G 2}=1535.5(\mathrm{~nm})\end{array}$ & $\begin{array}{l}v_{F P 2}=4.953(\text { cycles/nm) (Eq. (2)) } \\
\Delta_{\lambda B G 2}=3.229 \text { (nm) (Eq. (3)) } \\
v_{B G 2}=1.238 \text { (cycles/nm) (Eq. (9)) }\end{array}$ \\
\hline Fabry-Perot sensor 3 (S3) & $\begin{array}{l}L_{\mathrm{FP} 3}=4(\mathrm{~mm}) \\
L_{\mathrm{BG}}=0.5(\mathrm{~mm}) \\
n=1.46 \\
\lambda_{B G 3}=1539.5(\mathrm{~nm})\end{array}$ & $\begin{array}{l}v_{F P 3}=4.928(\text { ciclos/nm) (Eq. (2)) } \\
\Delta_{\lambda B G 3}=3.246(\mathrm{~nm}) \text { (Eq. (3)) } \\
v_{B G 3}=1.232(\text { cycles/nm) (Eq. (9)) }\end{array}$ \\
\hline
\end{tabular}

Table 2. Quasi-distributed sensor parameters used and signal values obtained.

$\Delta \lambda_{B G 3}=3.246(\mathrm{~nm})$. Finally, the triangle functions have approximately the same bandwidth: $v_{B G 1}=1.244$ (cycles $\left./ \mathrm{nm}\right), v_{B G 2}=1.238($ cycles $/ \mathrm{nm})$ and $v_{B G 3}=1.232$ (cycles $\left./ \mathrm{nm}\right)$. Thus, we prove that the quasi-distributed sensor applies the wavelength-division multiplexing and the FDM technique was eliminated.

Figure 7 Shows the behavior of demodulation error vs. signal-to-noise rate $\mathrm{SNR}^{1 / 2}$, if demodulation error is denominated as the resolution. These results confirm good resolution for the twin-grating sensors. Since the FDPA algorithm makes two evaluations of the Bragg wavelength shift $[3,7]$, the twin-grating sensors have two resolutions: Low resolution $\sigma_{\text {env }}$ and high resolution $\sigma_{\text {int }}$. the threshold [3].

$$
\sigma_{\mathrm{env}}<\frac{\lambda_{B G k}^{2}}{12 n L_{F P}}
$$

specifies the boundary between low resolution and high resolution. Using Eq. (29) and Table 2, the thresholds are approximately S1 $\rightarrow \sigma_{\text {env }}<0.0335 \mathrm{~nm}, \mathrm{~S} 2 \rightarrow \sigma_{\text {env }}<0.0336 \mathrm{~nm}$, and S3 $\rightarrow$ $\sigma_{\text {env }}<0.0338 \mathrm{~nm}$. For each local twin-grating sensor, an $\mathrm{SNR}^{1 / 2}$ threshold is observable:

$S 1(1531.5 \mathrm{~nm}) \rightarrow S N R^{\frac{1}{2}} \approx 10^{1.15}, S 2(1355.5 \mathrm{~nm}) \rightarrow S N R^{\frac{1}{2}} \approx 10^{1.19}, S 3(1539.5 \mathrm{~nm}) \rightarrow S N R^{1 / 2} \approx 10^{1.2}$. The threshold values are very close because the twin-grating sensors have similar cavity length and the enveloped functions have approximately the same bandwidth $\Delta \lambda_{B G k}$.

\subsection{Discussion}

The quasi-distributed fiber optic sensor (Figure 3) would be built on wavelength-division multiplexing and twin-grating interferometers. Our results optimize the sensor's implementation and also permit its design. Local sensor properties, light source characteristics, noise (Gaussian distribution), signal processing and detection technique are considered in our 
numerical simulation. Our experimental results (Figure 7) corroborate well functionally. Two resolutions are also confirmed $\sigma_{B G}$ for each local sensor: low resolution $\sigma_{\text {env }}$ and high resolution $\sigma_{\text {int }}$. Both resolutions depend on noise system, cavity length, instrumentation, sensor properties, and the digital demodulation algorithm.

A twin-grating fiber optic sensor and an optical fiber sensor based on a single Bragg grating will have the same resolution if and only if FDPA algorithm cannot eliminate the $2 \pi P$ ambiguity. In this case, the twin-grating sensor has only low resolution because the FDPA algorithm evaluates the Bragg wavelength shift with the enveloped function [11]. However, if the optical sensing system has small noise, then the twin-grating sensor has high resolution, since the Bragg wavelength shift was evaluated combining the enveloped and modulated functions $[3,11]$. Additionally, three twin-grating sensors have approximately the same resolution because three wavelength channels are very close and all interferometer sensors have the same cavity length. In conclusion, the sensor's resolution is high while the FDPA algorithm can acceptably demodulate the optical signal.

The presented study optimizes the quasi-distributed sensor which was shown in Figure 3. Combining our study (this work) and the analysis presented in Ref. [3], the experimental sensing system described by Shlyagin et al. [4,5] can be optimized. The optimization will be on signal processing, local sensor properties, sensitivity, resolution and instrumentation parameters. Additionally, the cost per sensing point is considerably reduced.

Our future work has the following direction: a theoretical analysis and practical application. In the theoretical analysis, frequency-and-wavelength division multiplexing can be implemented based on the twin-grating interferometer; resolution is another direction. In the practical

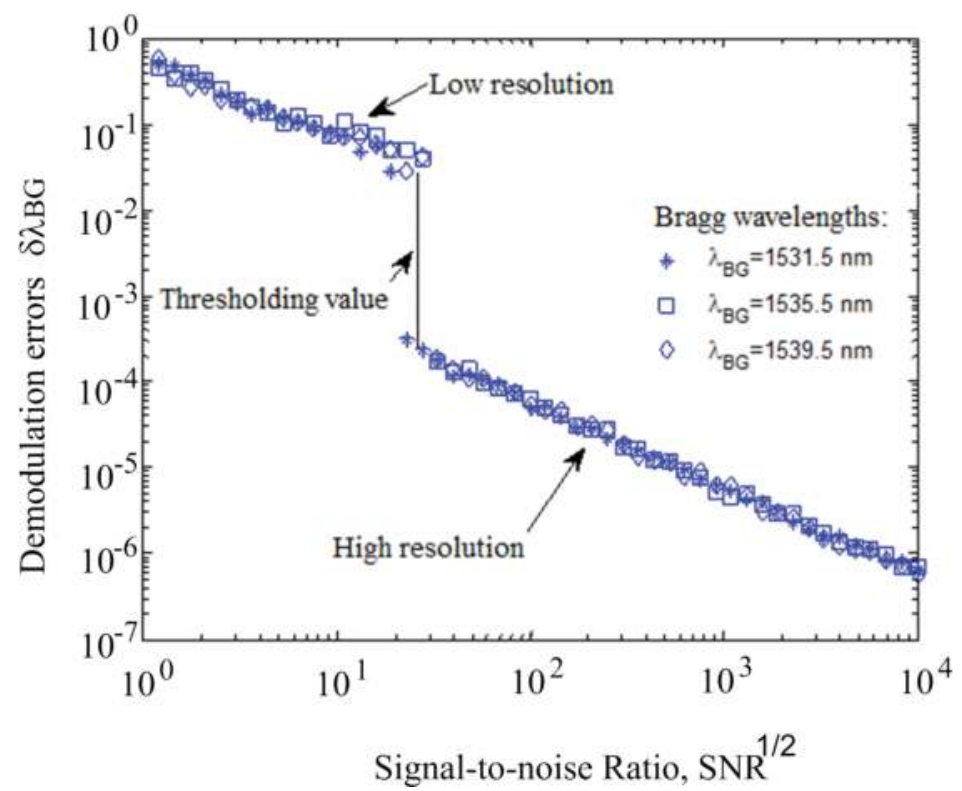

Figure 7. Numerical results obtained from the numerical experiments. 
applications, the quasi-distributed sensor can be applied for temperature monitoring, gasoline detection (security), strain measurement, and level liquid measurement. Our analysis makes an excellent contribution to quasi-distributed sensor implementation because all local sensors will have high resolution (see Figure 7), high sensibility, low cost by sensing point, and the quasi-distributed sensor can be designed without other requirements.

\section{Conclusion}

In this work, a quasi-distributed fiber optic sensor was numerically simulated. The sensor was based on twin-grating sensors and wavelength-division multiplexing. The numerical results show the resolution for each local twin-grating sensor. Local sensors have approximately the same resolution because all twin-grating sensors have the same cavity length and the wavelength channels are close. Two resolutions were obtained for each local sensor. Our numerical results show that the quasi-distributed sensor has potential industrial application: temperature measurement, strain measurement, pressure measurement, humidity monitoring, and security system.

\section{Acknowledgements}

Authors thank PRODEP 2017 No. F-PROMEP-39/Rev-04 SEP-23-005 (number DSA/103.5/16/ 10313), PRODEP 2017 Project No. 236110 of found 1.1.9.25 (Agreement RG/003/2017) and PRODEP 2017 Project No 238635 (511-6/17-8091).

\section{Author details}

José Trinidad Guillen Bonilla ${ }^{1,2 *}$, Héctor Guillen Bonilla ${ }^{3}$, Antonio Casillas Zamora ${ }^{1}$, Gustavo Adolfo Vega Gómez ${ }^{1}$, Nancy Elizabeth Franco Rodríguez ${ }^{4}$, Alex Guillen Bonilla ${ }^{5}$ and Juan Reyes Gómez

*Address all correspondence to: trinidad.guillen@academicos.udg.mx

1 Electronic Department, CUCEI, University of Guadalajara, Guadalajara, Jalisco, México

2 Mathematics Department, CUCEI, University of Guadalajara, Guadalajara, Jalisco, México

3 Department of Engineering Projects, CUCEI, University of Guadalajara, Guadalajara, Jalisco, México

4 Computer Department, CUCEI, University of Guadalajara, Guadalajara, Jalisco, México

5 Department of Computer Science and Engineering, CUValles, University of Guadalajara, Ameca, Jalisco, México

6 Faculty of Chemical Science, Colima University, Coquimatlán, Colima, Mexico 


\section{References}

[1] Kashyap R. Photosensitive optical fibers: Device and applications. Optical Fibre Technology. 1994;1:17-34

[2] Grattan KTV, Sun DT. Fiber optic sensor technology: An overview. Sensors and Actuators. 2000;82:40-61

[3] Guillen Bonilla JT, Guillen Bonilla A, Rodríguez Betancourtt VM, Guillen Bonilla H, Casillas Zamora A. A theoretical study and numerical simulation of a quasi-distributed sensor based on low-finesse Fabry-Perot interferometer: Frequency-division multiplexing. Sensors. 2017;17:859. DOI: 10.3390/s17040859

[4] Shlyagin MG, Miridonov SV, Márquez-Borbón I, Spirin VV, Swart PL, Chtcherbakov AA. Multiplexed twin Bragg grating interferometer sensor. In: Proceedings of the Optical Fiber Sensors Conference Technical Digest (OFS 2002); 10 May 2002; Portland, OR, USA. pp. 191-194

[5] Shlyagin MG, Miridonov SV, Tentori Santa-Cruz D, Mendieta Jiménez FJ, Spirin VV. Multiplexing of grating-based fiber sensors using broadband spectral coding. In: Proceedings of the Conference on Fiber Optic and Laser Sensors and Applications; 1 November 1998; Boston, MA, USA; Volume 3541, pp. 271-277

[6] Cibula E, Donlagic D. In-line short cavity Fabry-Perot strain sensor for quasi-distributed measurement utilizing standard OTDR. Optics Express. 2007;15(14):8728

[7] Yu XJ, Zhang YL, Li K, Zhang JT, Lv HJ, Liu SC. Frequency-division multiplexing sensing system based on multilongitudinal mode fiber lasers and beat frequency demodulation. IEEE Photonics Journal. 2015;7(2):7. ID: 7901307. DOI: 10.1109/JPHOT. 2015.2418265

[8] Wang Y, Gong J, Wang DY, Dong B, Bi W, Wang A. A quasi-distributed sensing network with time-division-multiplexing fiber Bragg gratings. IEEE Photonics Technology Letters. 2011;23(2):70-72. DOI: 10.1109/LPT.2010.2089676

[9] Yuan L, Zhou L, Jin W, Yang J. Design of a fiber-optic quasi-distributed strain sensors ring network based on a white-light interferometric multiplexing technique. Applied Optics. 2002;41(34):7205-7211. DOI: 10.1364/AO.41.007205

[10] Martinez-Manuel R, Shlyagin MG, Miridonov SV, Meyer J. Vibration disturbance localization using a serial array of identical low-finesse fiber Fabry-Perot interferometers. IEEE Sensors. 2012;12(1):124-127. DOI: 10.1109/JSEN.2011.2119479

[11] Miridonov SV, Shlyaing MG, Tentori D. Twin-grating fiber optic sensor demodulation. Optics Communication. 2001;191:253-262 
[12] Miridonov SV, Shlyagin MG, Tentori D. Digital demodulation of twin-grating fiber-optic sensor. In: Proceedings of the Conference on Fiber Optics and Laser Sensors and Applications, Boston, MA, USA; 1 November 1998; Volume 3541; pp. 33-40

[13] Miridonov SV, Shlyagin MG, Spirin VV. Resolution limits and efficient signal processing for fiber optic Bragg grating with direct spectroscopic detection. In: Proceedings of the Conference on Optical Measurement Systems for Industrial Inspection III, Munich, Germany; 2003; Volume 5144; pp. 679-686 
